\title{
Bataille en vue autour de la dette européenne : le projet annulationniste face à ses détracteurs
}

Pierre Pénet

Chargé de recherche CNRS

ENS Paris-Saclay

Quel avenir pour la dette publique ? Tribunes et prises de position se multiplient depuis le début de la pandémie pour recommander des mesures d'annulation de dette. Ces débats ne sont pas nouveaux : régulièrement s'ouvrent et se ferment des controverses sur la dette, les rapports entre États et créanciers et les moyens qu'ont les premiers d'échapper à l'emprise des seconds. Jusqu'à récemment cantonnées aux pays dits «émergents » ou «en développement », ces controverses sont désormais installées au cœur de l'Europe, où les politiques de lutte contre la pandémie ont fait croître l'endettement public à des niveaux jamais observés depuis 1945.

Le recours massif aux déficits pour financer les hôpitaux, le chômage partiel et les aides aux entreprises a dissipé certains dogmes budgétaires tenaces. Les critères de Maastricht ont été suspendus pendant que l'Allemagne mettait entre parenthèses sa règle d'or et que la France encourageait le «quoi qu'il en coûte». Cette séquence d'endettement, inédite par son intensité, a mis au centre du jeu politique la Banque centrale européenne. Suite aux mesures «non conventionnelles » de rachat des titres souverains, la BCE détient aujourd'hui $30 \%$ de la dette des États européens (contre seulement 5\% en 2015). L'absorption par les autorités monétaires de la majorité des émissions de dette publique a transformé l'identité des détenteurs de la dette et changé la nature même de l'endettement. Ces inflexions ouvrent des perspectives nouvelles en matière de politique de gestion de la dette : des voix se sont ainsi élevées récemment pour demander un traitement lui aussi non conventionnel et « hors cadre » de la dette accumulée, afin d'éviter que l'objectif du désendettement ne serve une nouvelle fois à cautionner une vague d'austérité désastreuse pour l'économie et les populations.

Parmi les mesures envisagées figurent l'annulation de la dette publique détenue à l'actif de la BCE. Cette proposition, soutenue par une centaine d'économistes ainsi que des responsables politiques, présente des aspects novateurs par rapport aux pratiques habituelles d'annulation. D'un point de vue socio-historique, il apparaît judicieux de prendre du recul et de situer cette initiative dans la longue histoire des annulations de dettes publiques. On va le voir, son originalité réside moins dans l'annulation en tant que telle que dans la proposition d'échanger et de convertir les montants de dette annulés en investissements dans la relance économique et la transition écologique. Séduisante en théorie, une telle négociation diplomatique autour de la dette pose cependant des questions ardues sur le plan politique, comptable et juridique.

Sans surprise, le projet «annulationniste » suscite de fortes résistances chez de nombreux économistes et responsables institutionnels qui prônent, outre le retour à l'austérité, l'ingénierie du cantonnement pour rembourser les dettes sur le temps long. Mais le débat ne se limite pas à une opposition entre orthodoxes et hétérodoxes. L'effacement d'une partie de la dette publique trouve également de nombreux contradicteurs chez des économistes classés à gauche qui proposent, en lieu d'une annulation, de recréer un pôle bancaire public pour sortir l'État des logiques de financement par le marché. Ce désaccord au sein du camp hétérodoxe traduit moins une divergence de fond sur la situation macroéconomique qu'une opposition tactique sur la meilleure façon d'exploiter la fenêtre d'opportunité politique ouverte par la pandémie. Ces échanges parfois véhéments expriment aussi un conflit d'ordre 
mémoriel, puisque les parties en présence convoquent des références historiques différentes pour justifier leurs positions.

La controverse sur l'annulation de la dette présente donc une valeur heuristique certaine. Elle permet tout d'abord d'identifier la gamme des alternatives qui pourraient faire obstacle à la réaffirmation de la doxa financière et budgétaire dans le contexte de l'après-Covid. Ensuite, et indépendamment du succès que ces propositions rencontreront, ce débat mérite également d'être étudié en lui-même car il donne accès aux imaginaires économiques et politiques parfois concurrents qui coexistent au sein du camp hétérodoxe.

\section{La diplomatie de l'annulation : convertir la dette publique en investissements}

Dans une note publiée en avril 2020, deux économistes de l'université de Nanterre, Laurence Scialom et Baptiste Bridonneau, proposent d'annuler une partie de la dette publique européenne $^{1}$. Reprise, en février 2021, dans une tribune largement commentée et signée par 150 économistes d'une dizaine de pays européens, la proposition repose sur une opération en deux temps : effacer les titres de dette publique détenus à l'actif de la BCE, en échange de l'engagement des États à investir un montant équivalent dans la relance économique et la transition écologique ${ }^{2}$. La BCE détenant $30 \%$ des créances de l'union monétaire, le montant de dette susceptible d'être converti en investissements s'élève à 2500 milliards d'euros (600 milliards en France) $)^{3}$.

La proposition a de quoi surprendre car elle sort des registres habituels d'annulation de dette. Il ne s'agit pas d'une annulation motivée par le constat d'une dette insoutenable, comme dans les cas récents de l'Argentine ou de l'Équateur. Malgré un haut niveau d'endettement, les pays européens ne sont pas au bord de la banqueroute et conservent la confiance des marchés. La proposition n'est pas non plus justifiée par l'urgence morale de réduire une dette publique illégitime, comme en Irak où le critère de la dette odieuse a été invoqué pour effacer des dettes contractées par un régime despotique ${ }^{4}$. Pour les signataires de la tribune, l'urgence n'est pas financière ou morale mais politique. L'annulation des dettes détenues par la BCE est envisagée comme une monnaie d'échange dans le cadre d'une négociation européenne visant à impulser des plans ambitieux de relance économique et d'investissement dans les politiques environnementales.

Dans son esprit, l'initiative évoque des accords négociés dans les années 1940-1950 autorisant des effacements de dette publique en échange d'investissements massifs dans l'économie et l'industrie. En 1942, un accord signé entre les États-Unis et le Mexique conduit les banques américaines à réduire de $90 \%$ leurs créances mexicaines. En contrepartie, le Mexique s'engage à contribuer à l'effort de guerre en investissant dans les infrastructures de transport, l'industrie pétrolière et la construction navale. Un sort similaire est réservé à la dette allemande après 1945. Les États-Unis lancent un vaste plan d'investissement en Allemagne en

\footnotetext{
1 «Des annulations de dettes publiques par la BCE : lançons le débat», Terra Nova, 18 avril 2020. Une proposition analogue a également été présentée en février 2020 dans l'ouvrage d'Alain Grandjean et de Nicolas Dufrêne (Une monnaie écologique, Paris, Odile Jacob, 2020).

2 «L'annulation des dettes publiques que la BCE détient constituerait un premier signal fort de la reconquête par l'Europe de son destin », Le Monde, 5 février 2021. Parmi les signataires : Jézabel Couppey-Soubeyran, Gaël Giraud, Thomas Piketty et Éric Toussaint. Jean-Luc Mélenchon, Benoit Hamon et Arnaud Montebourg ont apporté leur soutien.

${ }^{3}$ En pratique, ce sont les 27 banques centrales nationales de l'Eurosystème (piloté par la BCE) qui détiennent l'essentiel de la dette de leur pays.

${ }^{4}$ Pierre Pénet, « Rethinking Odious Debt », Books \& Ideas, 19 mars 2018.
} 
contrepartie d'une suspension du paiement de la dette, qui sera finalement réduite de moitié à l'Accord de Londres de 1953, au grand dam des investisseurs mais aussi de certains états créanciers, comme l'Angleterre, qui réclament le paiement complet des dettes d'avant-guerre. Ces deux cas sont représentatifs d'une forme de diplomatie financière qui relègue le remboursement de la dette au second plan par rapport à des considérations jugées prioritaires comme l'effort de guerre ou le redressement économique européen ${ }^{5}$.

Ces précédents historiques rappellent que le remboursement de la dette peut être subordonné à la réalisation d'objectifs politiques ou stratégiques. On trouve une prémisse analogue dans la proposition d'utiliser la dette publique européenne comme levier politique pour lancer des programmes d'investissement. Après des années de sous-investissement chronique, les besoins européens dans le domaine de l'énergie, des transports et du changement climatique sont chiffrés par la Cour des comptes européenne à 1115 milliards par an jusqu'à $2030^{6}$, un montant très supérieur aux sommes qui pourraient être dégagées par l'impôt, ou par l'outil budgétaire en raison des critères de Maastricht qui limitent la dette et le déficit publics à $60 \%$ et $3 \%$ du PIB. Ces critères, qui ont été suspendus durant la pandémie, s'appliqueront de nouveau en 2023. Malgré les critiques qui s'intensifient pour réclamer leur abandon, ces critères servent toujours de référence à Bercy qui envisage une cure d'austérité pour faire revenir le déficit à $2,8 \%$ du PIB en $2027^{7}$. Compte tenu des contraintes spécifiques à la zone euro, le projet d'annulation de la dette est présenté par ses promoteurs comme le seul permettant aux États européens d'engager des montants (2 500 milliards d'euros) à la hauteur des enjeux macroéconomiques de l'après-crise.

Le cadre institutionnel de la zone euro apparaît comme le facteur déterminant du débat sur la dette, un débat dont on remarque qu'il n'a pas cours aux États-Unis, où aucun verrou budgétaire ne fait obstacle à l'adoption de programmes ambitieux. Dans le sillage du gigantesque plan de relance voté en mars 2021 (1 900 milliards de dollars), la nouvelle administration Biden envisage désormais un programme d'investissement de 2300 milliards de dollars dans les infrastructures et les énergies propres. Alors qu'aux États-Unis la disponibilité de l'instrument budgétaire permet de telles politiques volontaristes, le verrou budgétaire européen crée un dilemme hirschmanien entre exit, voice et loyalty. Sauf à envisager de quitter la zone euro (exit) - une option remarquablement absente des débats -, ou à interpeller les institutions européennes en proposant un correctif (voice), l'acceptation du statu quo budgétaire (loyalty) rend impossible l'adoption de plans comparables en Europe.

L'initiative annulationniste appartient au registre de l'interpellation: elle subvertit une convention bien ancrée dans les mentalités qui associe l'extinction d'une dette avec son repaiement en rappelant que certaines dettes n'appellent pas nécessairement un remboursement et peuvent être mises en équivalence avec la réalisation d'objectifs politiques ou stratégiques. Cette interpellation produit indéniablement certains effets « libérateurs » dans le débat européen. Mais ses contradicteurs ont relevé plusieurs zones d'ambiguïté sur le plan juridique, comptable et financier.

\section{Réactiver une rhétorique conservatrice de la dette}

\footnotetext{
${ }^{5}$ Pierre Pénet et Juan Flores Zendejas (dir.), Sovereign Debt Diplomacies: Rethinking Sovereign Debt from Colonial Empires to Hegemony, Oxford, Oxford University Press, 2021.

${ }^{6}$ Cour des comptes européenne, «L'action de l'UE dans le domaine de l'énergie et du changement climatique », Analyse panoramique, 2017.

${ }^{7}$ «Bercy annonce une cure d'austérité pour les 5 prochaines années », L'Humanité, 9 avril 2021.
} 
La proposition annulationniste a fait l'objet de réactions scandalisées de la part d'économistes la décrivant comme une «idée idiote » (Olivier Blanchard) ou une pratique « vaudoue » (Jean Pisani Ferry $)^{8}$. Il est frappant de voir que chez ces économistes la proposition est jugée irrecevable avant même d'avoir été évaluée. Il en va de même chez certains responsables politiques de la majorité jusqu'à Marine Le Pen qui se bornent à invoquer le devoir «moral » des États de rembourser leurs dettes ${ }^{9}$. Au-delà de ces réactions instinctives, les débats qui se nouent autour du projet d'annulation sont surtout l'occasion de réactiver une rhétorique conservatrice de la dette. Pierre Moscovici, le Premier président de la Cour des comptes, s'inquiète ainsi pour les prochaines générations «qui auront à payer pour l'éternité une dette écrasante ${ }^{10}$. On reconnaît ici une dramaturgie bien rodée visant à décourager l'expression de toute proposition alternative. Ces récits ignorent pourtant que la France ne connaît actuellement aucun problème pour financer sa dette. L'État français emprunte à des taux historiquement faibles voire négatifs et peut, à la différence d'un ménage ou d'une entreprise, faire rouler sa dette, c'est-à-dire émettre de nouveaux emprunts lorsque les précédents arrivent à échéance. Ces discours oublient aussi le rôle des cadeaux fiscaux aux grandes entreprises et aux plus riches dans la dynamique de l'endettement. Les baisses d'impôts (45 milliards de recettes en moins depuis 2017) ont creusé les déficits sans effet notable sur l'emploi ou l'investissement; sans parler de l'évasion fiscale qui représente chaque année un énorme manque à gagner pour l'État.

La proposition d'un cantonnement de la dette Covid, estimée à 215 milliards d'euros, est une bonne synthèse de ces non-dits. L'ingénierie du cantonnement consiste à séparer une dette du reste du budget pour la placer dans une caisse alimentée par des ressources dédiées ${ }^{11}$. Isoler la dette Covid et étaler son remboursement sur le long terme permettrait de faire repasser le ratio dette-PIB sous le seuil psychologique de $100 \%$, mais la manœuvre serait purement cosmétique car la qualité de la signature de l'État sur les marchés financiers n'est pas menacée. Surtout, et contrairement au projet annulationniste, elle n'ouvre aucune perspective macroéconomique et ne permet pas de sortir de la problématique de l'endettement. Elle est au contraire un moyen de sanctuariser la dette et d'asseoir plus encore les finances publiques dans des contraintes disciplinaires ${ }^{12}$. La commission Arthuis pour l'avenir des finances publiques s'inscrit dans le même imaginaire conservateur : son rapport, publié en mars 2021, comporte des projections délibérément catastrophistes sur la dette (annoncée à 133\% du PIB en 2030) pour justifier la consolidation budgétaire et l'encadrement de la croissance des dépenses publiques dans une limite fixée à $0,65 \%$ du $\mathrm{PIB}^{13}$.

La solution du cantonnement et le rapport Arthuis témoignent avant tout d'une volonté d'un retour à la normale. Derrière les discours de façade sur la relance et l'investissement, c'est bien le retour de l'austérité qui se profile avec de nouvelles coupes attendues dans les prestations sociales et les systèmes de retraite pour «assainir » les comptes publics. C'est là que réside la principale différence avec les annulateurs qui estiment, quant à eux, que la distribution massive d'argent public durant la pandémie interdit tout retour aux méthodes anachroniques de la consolidation budgétaire.

\footnotetext{
${ }^{8}$ «Faut-il annuler la dette ? Les clés d'un débat qui divise les économistes », La Croix, 28 février 2021.

9 «Ayons des idées claires et du bon sens sur la dette », l'Opinion, 21 février 2021.

10 «Depuis le Covid, la dette publique a augmenté de 6500 euros par Français », Le Parisien, 3 octobre 2020.

${ }^{11}$ Un précédent est la création en 1996 de la Caisse d'amortissement de la dette sociale pour apurer les dettes du régime général de la sécurité sociale. La Cades émet des emprunts garantis par les revenus qui lui sont alloués : la contribution de remboursement de la dette sociale et une partie de la contribution sociale généralisée.

${ }^{12}$ Anne-Laure Delatte et Benjamin Lemoine, «Expertise économique et politique publique : examen critique des propositions sur la dette liée à la pandémie », LIEPP Working Paper, n 118, mars 2021.

${ }^{13}$ Henri Sterdyniak, « Rapport Arthuis ? Non, rien », Les Économistes Atterrés, 23 mars 2021.
} 
Malgré les critiques, la proposition annulationniste conserve une charge subversive. En témoignent les commentaires abondants qu'elle a suscités chez les banquiers centraux qui ont cherché à neutraliser le débat sur le terrain juridique. Christine Lagarde, présidente de la BCE, estime que le financement direct des États par un effacement de dette n'est pas prévu dans le mandat de la $\mathrm{BCE}^{14}$. S'il est exact que le terme annulation ne figure pas dans les traités, celui d'assouplissement quantitatif n'y figure pas non plus, ce qui n'a pas empêché ces politiques non-conventionnelles de devenir l'instrument central de la BCE durant la pandémie. Ces évolutions récentes sont l'occasion de rappeler que l'histoire des politiques monétaires sur le temps long déjoue toute posture légaliste et que les banques centrales ont toujours su faire évoluer leurs pratiques au gré des circonstances et indépendamment des cadres établis, que ce soit en matière de fixation des taux d'intérêt, de choix des garanties éligibles en contrepartie des opérations de crédit ou de mesures de recapitalisation bancaire ${ }^{15}$.

Il pourrait en aller de même, en théorie, de la gestion du portefeuille de titres de dette publique détenus à l'actif de la BCE. Qu'elle prenne la forme d'un abandon ou d'un effacement de créances, l'initiative annulationniste ne rencontre pas d'obstacle technique ou juridique insurmontable. Transformer la dette publique en dette perpétuelle, sans intérêt et sans exigence de remboursement connaît des précédents historiques. En revanche, l'option de l'effacement volontaire de la dette BCE est dépourvue de jurisprudence. Mais ce scénario, qui conduirait la banque centrale à enregistrer des pertes sur son bilan, n'est pas non plus inenvisageable dans la mesure où la $\mathrm{BCE}$ n'est soumise, en tant qu'institution publique, à aucune contrainte de rentabilité ou de liquidité et peut fonctionner avec des fonds propres négatifs ${ }^{16}$.

\section{Le camp hétérodoxe face à un désaccord tactique}

À côté de l'initiative annulationniste, d'autres propositions ont émergé pour contrer le retour annoncé à la doxa financière et budgétaire après la pandémie. Dans une tribune parue en février 2021, 80 universitaires proposent de sortir des logiques de financement de l'État par les marchés ${ }^{17}$. L'initiative consiste à financer directement les déficits en réactivant un « circuit du Trésor », une émanation de l'État bancaire de l'après-guerre démantelée dans les années $1970^{18}$. Ce procédé permettait au Trésor public de capter les liquidités des organismes publics ainsi que l'épargne privée collectée par les banques de dépôt. L'État percevait ainsi des ressources régulières pour investir dans les secteurs jugés nécessaires et stratégiques ${ }^{19}$. Le rétablissement de cet arrangement permettrait de financer des investissements ambitieux dans l'économie et la transition écologique sans être exposé au jugement des marchés et des agences de notation.

\footnotetext{
14 «Une annulation de dette est inenvisageable pour Christine Lagarde », JDD, 7 février 2021.

15 Il n'y a qu'à se rappeler les violentes controverses juridiques et comptables provoquées par les premières opérations d'open market conduites par la Réserve fédérale de New York durant les années 1920. Voir Allan Meltzer, A History of the Federal Reserve, Vol. 1 1913-1951, Chicago, University of Chicago Press, 2003.

${ }^{16}$ David Archer et Paul Moser-Boehm, «Central Bank Finances », BIS Papers, ${ }^{\circ}$ 71, avril 2013.

17 «D'autres solutions que l'annulation de la dette existent pour garantir un financement stable et pérenne », Le Monde, 27 février 2021. Parmi les signataires : Benjamin Lemoine, Éric Monnet, Thomas Porcher et Adam Tooze.

${ }^{18}$ Benjamin Lemoine, L'ordre de la dette : Enquête sur les infortunes de l'État et la prospérité du marché, Paris, La découverte, 2016.

${ }^{19}$ Éric Monnet, Controlling Credit: Central Banking and the Planned Economy in Postwar France, 1948-1973, Cambridge, Cambridge University Press, 2018.
} 
La reconstruction d'un pôle bancaire public est présentée comme une alternative puissante à la rhétorique conservatrice de la dette et aux dogmes budgétaires. Mais les défenseurs de cette alternative adressent aussi une critique sévère au projet annulationniste, le jugeant inutile ${ }^{20}$ ou dangereux ${ }^{21}$. Au-delà de ces différends, l'aspect le plus intéressant de cet échange est sans doute de nature tactique. Le débat entre universitaires hétérodoxes témoigne, en effet, d'un désaccord profond sur la meilleure façon d'exploiter la fenêtre d'opportunité politique ouverte par la pandémie. L'annulation de la dette BCE est une mesure ponctuelle ad hoc qui ne modifie pas les conditions d'endettement. La création d'un nouveau secteur bancaire public implique en revanche une transformation en profondeur - structurelle - des rapports Étatmarché, que ses promoteurs présentent comme la plus juste mesure du courage politique. La nature différente des changements envisagés inscrit les deux propositions dans des horizons temporels eux-aussi contrastés. Tandis que l'initiative annulationniste pourrait se concrétiser rapidement, car elle repose sur une opération comptable a priori simple sans modification des traités, la réorganisation du système d'endettement s'inscrit dans un processus législatif de plus long terme $\mathrm{e}^{22}$.

Un dernier point concerne l'écho des propositions dans le débat politique. De nouveau, la position annulationniste a l'avantage de ses défauts : moins subversive, car n'impliquant pas de changement structurel, cette proposition a l'avantage tactique de parler dans la langue de la doxa. Les investissements envisagés dans la transition écologique ne feront pas gonfler le ratio dette-PIB puisqu'ils sont financés en contrepartie de l'effacement de la dette BCE. On comprend ainsi dans quelle mesure certains acteurs politiques du centre ou de la droite seraient fondés à soutenir une initiative qui redonnerait des marges de manœuvre budgétaires aux États dans le cadre des institutions européennes existantes ${ }^{23}$. De son côté, et malgré les réticences affichées, la BCE pourrait y trouver l'occasion d'accéder aux demandes grandissantes d'élargir son mandat à la prise en compte des objectifs climatiques ${ }^{24}$. On peine en revanche à identifier les éléments qui persuaderaient les acteurs politiques et administratifs de rebâtir un système d'endettement public face à l'opposition farouche du secteur bancaire. Cela n'enlève en rien le mérite de la proposition, indéniablement plus ambitieuse que l'initiative annulationniste, mais c'est constater qu'il est plus difficile d'identifier ses conditions de félicité et d'envisager le cadre de négociation qui lui permettrait d'aboutir dans le champ politique européen actuel.

Ces aspects tactiques et stratégiques ne nous éloignent pas de la substance du débat, bien au contraire. Sociologues et historiens ont de longue date observé le poids des discours et des récits dans les modes de production et de reproduction des croyances économiques ${ }^{25}$. Face à la rhétorique conservatrice de l'endettement, toujours bien vivace, le succès des alternatives à

\footnotetext{
${ }^{20}$ L'annulation serait inutile car les taux bas donnent déjà aux États des marges budgétaires pour investir. Ce raisonnement, fondé en raison, occulte cependant les contraintes politiques qui ont conduit ces dernières années les États européens à se désendetter plutôt que de profiter des conditions favorables d'emprunt.

${ }^{21}$ Certains craignent que l'annulation envoie de mauvais signaux aux marchés et entraîne l'augmentation des taux d'emprunt. Ce scénario est pourtant peu probable car les créanciers privés sont exclus de l'opération et parce que la poursuite de l'activisme monétaire de la BCE et l'excès structurel d'épargne lié au vieillissement de la population vont maintenir durablement les taux bas.

${ }^{22}$ Il est à noter que ces deux temporalités sont présentées par les annulationnistes comme complémentaires, tandis que les opposants à l'annulation semblent refuser par principe des réformes ad hoc et de court terme.

${ }^{23}$ Des libéraux comme Alain Minc ou David Sassoli, le président du Parlement européen, ont apporté leur soutien.

${ }^{24}$ Voir Barry Eichengreen, « Les Banques centrales nouveau modèle », Project Syndicate, 9 février 2021.

${ }^{25}$ Frédéric Lebaron, La Croyance économique. Les économistes entre science et politique, Paris, Seuil, 2000.
} 
l'austérité passent par la mise en place de contre-discours évocateurs ${ }^{26}$. C'est tout particulièrement le cas en Europe où le respect de la discipline budgétaire continue d'être perçu comme indispensable à la survie de l'union économique et monétaire. De ce point de vue, et malgré ses zones d'ambiguïté, le discours annulationniste comporte plusieurs aspects subversifs. Il renverse les termes du débat en suggérant que l'avenir politique et économique de l'Europe passe par des programmes ambitieux de relance et d'investissement finançables en contrepartie de mesures d'effacement partiel de la dette des États. Cette diplomatie de la dette a, du reste, l'avantage d'être envisageable dans le cadre des institutions européennes, ce qui accroît ses chances d'atteindre des décideurs et des partis politiques prêts à défendre des politiques macroéconomiques volontaristes dans le contexte d'après-crise, mais qui souhaitent s'épargner un débat sur la sortie de la zone euro.

\footnotetext{
${ }^{26}$ À propos des aspects rhétoriques qui accompagnent les politiques ambitieuses du New Deal en matière de régulation bancaire, voir Pierre Pénet, «Rhetorical Metrics: Building Securities Regulation in America’s Era of Booms and Busts, 1890-1940 », European Journal of Sociology/Archives Européennes de Sociologie, vol. 60, $\mathrm{n}^{\circ} 1,2019$, p. 69-107.
} 